\title{
Solving regularized equations in the form of polynomials
}

\author{
Vera Petelina, ${ }^{1, *}$ \\ ${ }^{1}$ Moscow State University of Civil Engineering, Yaroslavskoye shosse, 26, Moscow, 129337, Russia
}

\begin{abstract}
The article is devoted to the determination of second-order perturbations in rectangular coordinates and components of the body motion to be under study. The main difficulty in solving this problem was the choice of a system of differential equations of perturbed motion, the coefficients of the projections of the perturbing acceleration are entire functions with respect to the independent regularizing variable. This circumstance allows constructing a unified algorithm for determining perturbations of the second and higher order in the form of finite polynomials with respect to some regularizing variables that are selected at each stage of approximation. The number of approximations is determined by the given accuracy. It is rigorously proven that the introduction of a new regularizing variable provides a representation of the right-hand sides of the system of differential equations of perturbed motion by finite polynomials. Special points are used to reduce the degree of approximating polynomials, as well as to choose regularizing variables.
\end{abstract}

\section{Introduction}

One of the crucial tasks associated with trajectory measurements is the determination of the partial derivatives of rectangular coordinates that make up the body motion speed with respect to the initial conditions. In operations [1-2] added auxiliary functions, which are degree series with respect to the auxiliary variable. In operations [3-5] outlined ways of using universal variables in a number of tasks of mechanics to determine disturbances by the method of variation of arbitrary constants. In this case, it is convenient to consider the components of the initial values of the radius-vector and velocity as osculating variables. New methods for determining disturbances keep the standard features of the classical ones, while calculating the disturbances, the small parameter method is used, which makes it possible to obtain asymptotic decomposition of the solution. Recently, Picard method for integration of differential equations is more commonly used, which leads to a convergent process of successive approximations that gives a solution to a system of differential equations. The error of the solution depends on the accuracy of the initial approximation of the perturbation function.

General principles of the development of perturbation theory in coordinates were analyzed in operations [6-8] studied the use of regularizing variables for calculation of trajectories of

\footnotetext{
${ }^{*}$ Corresponding author: verapetelina51@gmail.com
} 
motion in operation. The results of this research show that the use of regularizing variables increases the computer-based accuracy of calculations and significantly reduces the calculation time.

A crucial task of mechanics is to approximate the rectangular coordinates that make up the body speed and time in case of disturbed motion by algebraic polynomials of the lowest degree with respect to the auxiliary variable with a predetermined degree of accuracy.

One of the important problems in mechanics is the approximation of rectangular coordinates constituting the body velocity and time when the motion is perturbed by the lowest degree algebraic polynomials relative to the auxiliary variable with a predetermined degree of accuracy.

This paper describes a special system of differential equations of the perturbed moving body and this system is integrated through successive approximations method, which using the coordinates and constituents body velocity, take the form of polynomials in powers of some auxiliary variable. Its own independent variable is taken at each approximation step.

\section{Mathematical model}

In articles [9-11], there were obtained rectangular coordinates $x, y$ regularized speed components $x^{\prime}, y^{\prime}$ time $t$ as an initial approximation in the following form:

$$
\begin{gathered}
x=\frac{A_{1}}{w}+R_{x}(w) \cdot \ln w+R_{x 1}(w)+\varepsilon\left(R_{x 1}, n^{*}\right) \\
y=\frac{A_{2}}{w}+R_{y}(w) \cdot \ln w+R_{y 1}(w)+\varepsilon\left(R_{y 1}, n^{*}\right) \\
x^{\prime}=\frac{A_{3}}{w}+R_{x^{\prime}}(w) \cdot \ln w+R_{x^{\prime} 1}(w)+\varepsilon\left(R_{x^{\prime} 1}, n^{*}\right) \\
y^{\prime}=\frac{A_{4}}{w}+R_{y^{\prime}}(w) \cdot \ln w+R_{y^{\prime} 1}(w)+\varepsilon\left(R_{y^{\prime} 1}, n^{*}\right) \\
t=\frac{A_{5}}{w}+R_{t}(w) \cdot \ln w+R_{t 1}(w)+\varepsilon\left(R_{t 1}, n^{*}\right),
\end{gathered}
$$

where $R_{x}, R_{x 1}, R_{y}, R_{y 1}, R_{x^{\prime}}, R_{x^{\prime} 1}, R_{y^{\prime}}, R_{y^{\prime} 1}, R_{t}, R_{t 1}$ - polynomials in powers of the regularizing variable $w$. Let us prove that there are no terms of the form $\frac{1}{w}$ in the rectangular coordinates and time of the perturbed motion of the body (1).

The fundamental matrix of particular solutions of the equations in variations of the regularized equations of the 2-body problem is given by:

$$
D=\left(\begin{array}{cccc}
q_{1}^{(1)} & \cdot & \cdot & q_{1}^{(7)} \\
q_{2}^{(1)} & \cdot & \cdot & q_{2}^{(7)} \\
\cdot & \cdot & \cdot & \cdot \\
q_{7}^{(1)} & \cdot & \cdot & q_{7}^{(7)}
\end{array}\right)
$$

First order perturbations in auxiliary quantities $C_{i}$ are defined as follows [11]

$$
C_{i}=\int_{2}^{w}\left(\frac{D_{4 i}}{D} \cdot X+\frac{D_{5 i}}{D} \cdot Y+\frac{D_{6 i}}{D} \cdot Z\right) \cdot d w
$$

where $X, Y, Z$ - components of the perturbing acceleration due to the attraction of the body under study by the disturbing body, 


$$
\begin{gathered}
X=\frac{b_{-2}}{w^{2}}+\frac{b_{-1}}{w}+P_{1 n}(w)+\varepsilon\left(P_{1 n}, n\right) \\
Y=\frac{e_{-2}}{w^{2}}+\frac{e_{-1}}{w}+P_{2 n}(w)+\varepsilon\left(P_{2 n}, n\right) \\
Z=\frac{f_{-2}}{w^{2}}+\frac{f_{-1}}{w}+P_{3 n}(w)+\varepsilon\left(P_{3 n}, n\right),
\end{gathered}
$$

$P_{1 n}(w), P_{2 n}(w), P_{3 n}(w)$ - polynomials in powers of the regularizing variable $w$ to some degree $n, \varepsilon\left(P_{1 n}, n\right), \varepsilon\left(P_{2 n}, n\right), \varepsilon\left(P_{3 n}, n\right)$ - the errors of the corresponding approximations, which can be made arbitrarily small for large $n, D_{4 i}$ algebraic complement of an element, standing in the 4th row and in the $i$ matrix column (2), $D_{5 i}, D_{6 i}$ - introduced similarly, $D$ matrix determinant (2). Substituting expressions (4), (3) into the expressions for the rectangular coordinates and time given in article [11], we obtain

$$
\begin{gathered}
x=x_{0}+\sum_{l=1}^{7} q_{1}^{(l)} \cdot \int_{2}^{w}\left[\frac{D_{4 l}}{D} \cdot\left(\frac{b_{-2}}{w^{2}}+\frac{b_{-1}}{w}+P_{1 n}(w)+\varepsilon\left(P_{1 n}, n\right)\right)+\right. \\
\left.+\frac{D_{5 l}}{D} \cdot\left(\frac{e_{-2}}{w^{2}}+\frac{e_{-1}}{w}+P_{2 n}(w)+\varepsilon\left(P_{2 n}, n\right)\right)+\frac{D_{6 l}}{D} \cdot\left(\frac{f_{-2}}{w^{2}}+\frac{f_{-1}}{w}+P_{3 n}(w)+\varepsilon\left(P_{3 n}, n\right)\right)\right] \cdot d w= \\
=x_{0}+\sum_{l=1}^{7} q_{1}^{(l)} \cdot \int_{2}^{w}\left(\frac{D_{4 l}}{D} \cdot b_{-2}+\frac{D_{5 l}}{D} \cdot e_{-2}+\frac{D_{6 l}}{D} \cdot f_{-2}\right) \cdot \frac{1}{w^{2}} \cdot d w+R_{0, x}(w) \cdot \ln w+ \\
+R_{1, x}(w)+\varepsilon\left(R_{0, x} ; R_{1, x}\right)=x_{0}-\frac{1}{w} \cdot\left(b_{-2} \cdot \sum_{l=1}^{7} q_{1}^{(l)} \cdot \frac{D_{4 l}}{D}+e_{-2} \cdot \sum_{l=1}^{7} q_{1}^{(l)} \cdot \frac{D_{5 l}}{D}+\right. \\
\left.+f_{-2} \cdot \sum_{l=1}^{7} q_{1}^{(l)} \cdot \frac{D_{6 l}}{D}\right)+\tilde{R}_{0, x}(w) \cdot \ln w+\tilde{R}_{1, x}(w)+\varepsilon\left(\tilde{R}_{0, x} ; \tilde{R}_{1, x}\right),
\end{gathered}
$$

where $R_{0, x}(w), R_{1, x}(w), \tilde{R}_{0, x}(w), \tilde{R}_{1, x}(w)$ - polynomials in powers of the regularizing variable $w, \varepsilon\left(R_{0, x} ; R_{1, x}\right), \varepsilon\left(\tilde{R}_{0, x} ; \tilde{R}_{1, x}\right)$ - measure of inaccuracy for coordinate representation $x$ as an amount $\tilde{R}_{0, x}(w) \cdot \ln w$ and a polynomial in powers $w$. It will be similar for $y, z$, t. So, the coefficient of component $\frac{1}{w}$ in terms of (5) would be zero because as it is equal to the sum of works of first row elements of matrix determinant (2) on algebraic additions of other rows, which is what we set out to prove. If, as a first approximation, we consider the trajectory of close passage from the disturbing body, then the components of the perturbing acceleration $X, Y, Z$ have complex conjugate singular points $w=\alpha \pm i \beta$. Components $X, Y$, $Z$ are as follows [11]

$$
X=\frac{R_{n}(w)}{\left[(\alpha-w)^{2}+\beta^{2}\right]^{3 / 2}}
$$

where $R_{n}(w)$ - some polynomial with respect to the regularizing variable $w$ power of $n$. The components $Y$ and $Z$ have similar expressions. In order to determine the perturbations of the first and second order in rectangular coordinates and the components of the regularized velocity of the studied body, it is necessary to take the integral of functions (6). Let us introduce a new independent regularizing variable $v$ so as to represent the coordinates of the velocity and time in the first approximation for the trajectories of close passage from the disturbing body in the form of polynomials in powers of this variable

$$
w=\alpha+\beta \cdot s h v, d w=\beta \cdot c h v \cdot d v
$$


By performing the indicated replacement of an independent variable $w$, we obtain that the sought integral of function (6) has the form:

$$
\int \frac{R_{n}(s h v)}{\beta^{2} \cdot c h^{2} v} \cdot d v
$$

where $R_{n}(s h v)$ - a polynomial function in powers of $s h v$.

The integral (8) has the following structure

$$
\frac{1}{\beta^{2}} \cdot \int \frac{R_{n}(\operatorname{sh} v)}{c^{2} v} \cdot d v=l_{0} \cdot v+\frac{l_{1}}{c h v}+l_{2} \cdot t h v+\tilde{Q}(\operatorname{sh} v, \operatorname{ch} v),
$$

where $l_{0}, l_{1}, l_{2}$ - some factors, $\tilde{Q}(s h v, c h v)$ - a polynomial function in powers of $c h v$. We will show that this is true. Let us represent the polynomial $R_{n}(s h v)$ as follows

$$
R_{n}(s h v)=a_{0}+s h v \cdot R_{1}\left(\operatorname{ch}^{2} v\right)+\operatorname{ch}^{2} v \cdot R_{2}\left(\operatorname{ch}^{2} v\right),
$$

That is, from polynomial $R_{1}\left(\operatorname{ch}^{2} v\right)$ for odd degrees $s h v$, we take out the accumulation factor $s h v$, from the polynomial function $R_{2}\left(c h^{2} v\right)$ for even degrees $s h v$, we take out the accumulation factor $c h^{2} v$. Integrating, we shall obtain:

$$
\begin{gathered}
\left.\int \frac{s h v \cdot R_{1}\left(\operatorname{ch}^{2} v\right)}{c h^{2} v} \cdot d v=\int \frac{B_{0}}{c h^{2} v}+B_{2}+B_{4} \cdot \operatorname{ch}^{2} v+\cdots\right) \cdot d(\operatorname{ch} v)= \\
=-\frac{B_{0}}{c h v}+B_{2} \cdot \operatorname{ch} v+\frac{B_{4} \cdot c h^{3} v}{3}+\cdots+C_{1} \\
\int R_{2}\left(c h^{2} v\right) \cdot d v=\int\left(\frac{A_{0}}{c h^{2} v}+A_{2}+A_{4} \cdot \operatorname{ch}^{2} v+\cdots\right) \cdot d v= \\
=A_{0} \cdot t h v+A_{2} \cdot v+A_{4} \cdot \operatorname{sh} 2 v+\cdots+C_{2}
\end{gathered}
$$

Singular points $w=\alpha \pm i \beta$, which are branch points, are equivalent to those values of the independent regularizing variable $v$ for which $c h v=0, s h v= \pm i$, namely

$$
v=i \cdot\left(\frac{\pi}{2}+k \cdot \pi\right), k=0,1,2, \ldots
$$

We consider the integral of function (6) near a singular point $w=\alpha+i \beta$.

We introduce a new independent variable $\lambda$ in the following way:

$$
\lambda=w-(\alpha+i \beta)
$$

Using formula (7), we rewrite the expression for $c h v$ in the following form:

$$
\operatorname{ch} v=\sqrt{\left(1+s h^{2} v\right)}=\frac{\sqrt{\left((w-\alpha)^{2}+\beta^{2}\right)}}{\beta}
$$

From the formula (14), we obtain:

$$
w-\alpha=\lambda+i \beta
$$

We substitute expression (16) into expression (15), as a result we obtain:

$$
c h v=\frac{\sqrt{\lambda}}{\beta} \cdot \sqrt{(2 i \beta+\lambda)}
$$

The expression for $\operatorname{ch} v$ can be represented as the following factorization

$$
\operatorname{ch} v=\frac{\sqrt{\lambda}}{\sqrt{\beta}} \sqrt{2 i}+\frac{\sqrt{\lambda}}{\beta} \frac{1}{2}(2 i \beta)^{-\frac{1}{2}} \cdot \lambda+\frac{\frac{1}{2} \cdot\left(-\frac{1}{2}\right)}{2 !} \cdot(2 i \beta)^{-\frac{3}{2}} \lambda^{2} \cdot \sqrt{\frac{\lambda}{\beta^{2}}}+\cdots
$$

Singular point $\lambda=0$ is a branch point. 
Components of perturbing acceleration $X, Y, Z$ in the vicinity of a singular point $w=\alpha+i \beta$ are represented by factorizations of the following form:

$$
X=\frac{b_{-3 / 2}}{\lambda^{3 / 2}}+\frac{b_{-1 / 2}}{\lambda^{1 / 2}}+\lambda^{1 / 2} \cdot B(\lambda),
$$

where $B(\lambda)$ - a function without negative degree $\lambda$ because after replacing the independent regularizing variable $w$ by $v$, the components of the perturbing acceleration have the form:

$$
X=\frac{R_{n}(s h v)}{c h^{3} v}
$$

where $c h v$ is determined by factorizations (18). Perturbations in auxiliary quantities $C_{i}$ in a neighborhood of a singular point are defined as follows

$$
C_{i}=\int_{\lambda_{0}}^{\lambda}\left(\frac{D_{4 i}}{D} \cdot X+\frac{D_{5 i}}{D} \cdot Y+\frac{D_{6 i}}{D} \cdot Z\right) \cdot d \lambda,
$$

$(i=1, \ldots, 7)$,

where $D$ - a determinant of the fundamental matrix (2) of particular solutions of equations in variations of the regularized equations of the 2-body problem.

Representing $\frac{D_{4 i}}{D}, \frac{D_{5 i}}{D}, \frac{D_{6 i}}{D}$ in the form of polynomials in powers of a regularizing variable $\lambda$, we obtain expression (21) as follows

$$
C_{i}=\frac{e_{-0.5 i}}{\lambda^{0.5}}+T_{i}(\lambda)
$$

$(i=1, \ldots, 7)$,

where $T_{i}(\lambda)$ is a function without negative degrees $\lambda$,

$e_{-0.5 i}$ - certain coefficients.

Thus, in the vicinity of the singular point $w=\alpha+i \beta$ the coordinates and time of the perturbed motion of the body under study are represented by functions without negative degrees $\lambda$.

Let us define the value of the polynomial function $R_{n}(w)$ in the singular point $w=\alpha+i \beta$.

Substituting values $s h v= \pm i ; c h v=0$ into the expression (13), we obtain:

$$
R_{n}(s h v= \pm i)=a_{0}+i \cdot R_{1}(0)=a_{0}+i b_{0}=\delta_{1}
$$

where $\delta_{1}$ is a certain complex number.

The components of the perturbing acceleration $X, Y, Z$, taking into account expression (10), are representable in the form of factorizations for the neighborhood of points $s h v= \pm i$; $c h v=0$ in the form

$$
X=\frac{a_{0}}{c h^{2} v}+\frac{b_{0} \cdot s h v}{c h^{2} v}+\tilde{R}(c h v)
$$

where $\tilde{R}(c h v)$ - a polynomial in powers of $c h v$. Let us represent dependence $\frac{D_{4 i}}{D}, \frac{D_{5 i}}{D}, \frac{D_{6 i}}{D}$ in the form of polynomials in powers of $s h v$ :

$$
\frac{D_{4 i}}{D}(s h v)=c_{0}+d_{0} \cdot s h v+\operatorname{ch}^{2} v \cdot \tilde{T}(\operatorname{sh} v, \operatorname{ch} v),
$$


where $\tilde{T}(\operatorname{sh} v, c h v)$ - a function without negative degrees $c h v$. Let us define the value of the polynomial $\frac{D_{4 i}}{D}$ at points $v$, where $s h v= \pm i, \quad c h v=0$.

Let us draw an analogy between expressions (19) and (24). By comparison, we have the coefficient $b_{-\frac{3}{2}}=a_{0}+i b_{0}$.

Perturbations in auxiliary variables $C_{i}$ will have the following form

$$
C_{i}=\int\left(\frac{D_{4 i}}{D} \cdot X+\frac{D_{5 i}}{D} \cdot Y+\frac{D_{6 i}}{D} \cdot Z\right) d v
$$

Substituting expressions (24) and (25) into expressions (26), we have

$$
C_{i}=a_{1 i} \cdot t h v+a_{2 i} \cdot \frac{1}{c h v}+a_{3 i} \cdot v+\tilde{R}_{i}(s h v, c h v)
$$

$(i=1, \ldots, 7)$,

where $\tilde{R}_{i}(s h v, c h v)$ - a function without negative degrees $c h v$,

$a_{1 i}, a_{2 i}, a_{3 i}$ - certain coefficients.

Extending the above proof for a neighborhood of singular points to the general case, for any points of the complex plane $\mathrm{v}$, we have that the coordinates that make up the regularized velocity and time in the second approximation for the trajectories of close passage from the disturbing body are representable by polynomials with respect to the new regularizing variable $v$.

\section{Conclusions}

The article is devoted to the determination of second-order perturbations in rectangular coordinates and components of the body motion to be under study. The main difficulty in solving this problem was the choice of a system of differential equations of perturbed motion, the coefficients of the projections of the perturbing acceleration are entire functions with respect to the independent regularizing variable. This circumstance allows constructing a unified algorithm for determining perturbations of the second and higher order in the form of finite polynomials with respect to some regularizing variables that are selected at each stage of approximation. The number of approximations is determined by the given accuracy. It is rigorously proven that the introduction of a new regularizing variable provides a representation of the right-hand sides of the system of differential equations of perturbed motion by finite polynomials. Special points are used to reduce the degree of approximating polynomials, as well as to choose regularizing variables.

\section{References}

1) P. Xu, Comm. In Nonl. Sci. and Num. Sim., 59, 515-543 (2018)

doi:10.1016/j.cnsns.2017.11.021

2) K. Schiefermayr, Jour. of Math. Analysis and App., 445, 871-883 (2017)

doi:10.1016/j.jmaa.2016.08.021

3) Z. Fuqiang, H. Qingxue, X. Jiaquan, L. Yugui, M. Lifeng, W. Jianmei, App. Math. and Comp., 313, 321-330 (2017)

4) V. Lychagin, V. Yumaguzhin, Jour. of Geom. and Phys., 130, 213-228 (2018)

doi: 10.1016/j.geomphys.2018.03.025

5) Z. Wen, G. Gundersen, J. Heittokangas, Jour. of Differ. Equa., 264, 98-114 (2018)

doi:10.1016/j.jde.2017.09.003 
6) H. Tong, M. Ng, Jour. of Comp., 49, 85-94, (2018) doi.org/10.1016/j.jco.2018.08.001 7) N. Huu Can, N. Huy Tuan, V. Van Au, L. Duc Thang, Jour. of Math. Analysis and App., 462, 1148-1177 (2018) doi.org/10.1016/j.jmaa.2018.01.066

8) D. Torre, R.Flores, E.Fantino, Acta Astronautica, 153, 26-38 (2018) doi:10.1016/j.actaastro.2018.10.010

9) V. Petelina, MATEC, 196, 01016 (2018) doi:10.1051/matecconf $/ 201819601016$

10) V. Petelina, IOP Conf. Ser: Mater., 456, 012123 (2018) doi:10.1088/1757$899 \mathrm{X} / 456 / 1 / 012123$

11) V. Petelina, E3S Web of Conf. 110, 01031 (2019) doi:10/1051/e3sconf/201911001031 